\title{
SOBOLEV INEQUALITIES FOR PRODUCTS OF POWERS
}

\section{A. EDUARDO GATTO AND RICHARD L. WHEEDEN}

\begin{abstract}
We derive weighted Sobolev inequalities of the form $\|f\|_{L_{u}^{q}} \leq$ $C\|\nabla f\|_{L_{v}^{p}}, f \in C_{0}^{\infty}\left(\mathbf{R}^{n}\right), 1<p \leq q<\infty$, for classes of weight functions $u, v$ which include $v$ 's that are a finite product of certain power weights times an $A_{p}$ function.
\end{abstract}

\section{INTRODUCTION}

In this paper we derive sufficient conditions on a pair of weight functions $u, v$ in $\mathbf{R}^{n}, n>1$, so that the Sobolev inequality

$$
\left(\int_{\mathbf{R}^{n}}|f(x)|^{q} u(x) d x\right)^{1 / q} \leq c\left(\int_{\mathbf{R}^{n}}|\nabla f(x)|^{p} v(x) d x\right)^{1 / p}, \quad f \in C_{0}^{\infty},
$$

holds with $1<p \leq q<\infty$ and $c$ independent of $f$.

In order to put our results in some perspective, we recall a few known facts. We use the notation $u \in D^{\infty}$, and say that $u$ is a doubling weight, if $u(2 B) \leq$ $c u(B)$ for all balls $B \subset \mathbf{R}^{n}$, where $2 B$ denotes the ball concentric with $B$ whose radius is twice that of $B$, and $u(B)=\int_{B} u d x$. It is shown in [2] that (1.1) holds when $p<q$ if $u$ and $v$ are related by

$$
|B|^{1 / n} u(B)^{1 / q} \leq c v(B)^{1 / p}
$$

and if $u \in D^{\infty}$ and $v \in A_{p}:$ by $v \in A_{p}$, we mean that

$$
\left(\frac{1}{|B|} \int_{B} v d x\right)\left(\frac{1}{|B|} \int_{B} v^{-1 /(p-1)} d x\right)^{p-1} \leq c
$$

for all balls $B$. This result has been improved in [6] where it is shown that (1.1) holds when $p<q$ if $u, v^{-1 /(p-1)} \in D^{\infty}$ and

$$
|B|^{1 / n-1} u(B)^{1 / q}\left(\int_{B} v^{-1 /(p-1)} d x\right)^{1 / p^{\prime}} \leq c
$$

for all balls $B, 1 / p+1 / p^{\prime}=1$. It is also shown in [6] that (1.1) holds for $p \leq q$ without any doubling assumptions on the weights if there exists $r>1$

Received by the editors February 1, 1988.

1980 Mathematics Subject Classification (1985 Revision). Primary 46E35.

Supported in part by NSF Grant DMS 87-03546. 
such that

$$
|B|^{1 / n-1}\left[\left(\int_{B} u^{r} d x\right)^{1 / r}|B|^{1 / r^{\prime}}\right]^{1 / q}\left[\left(\int_{B} v^{-r /(p-1)} d x\right)^{1 / r}|B|^{1 / r^{\prime}}\right]^{1 / p^{\prime}} \leq c
$$

for all balls $B$. It follows easily from Hölder's inequality that this condition implies the previous one, which may be viewed as the case $r=1$.

These results have the disadvantage of requiring the local integrability of $v^{-1 /(p-1)}$. Thus, for example, the possibility that $v(x)$ might be a large positive power $|x|^{N}$ is precluded, while the results of [1 and 3] show that Sobolev inequalities do hold for appropriate $u$ if $v$ is an arbitrarily large power of $|x|$. The purpose of this paper is to give some further examples of weights $v$ for which $v^{-1 /(p-1)}$ is not locally integrable but for which (1.1) holds.

Our main results are given in the following two theorems and their corollaries.

Theorem (1.2). Let $\left\{a_{j}\right\}_{j=1}^{m}$ be distinct points in $\mathbf{R}^{n},\left\{\alpha_{j}\right\}_{j=1}^{m}$ and $\delta$ be real numbers, $\alpha_{j}>-1, \delta>-1$, and let

$$
\Pi(x)=(1+|x|)^{\delta} \prod_{j=1}^{m}\left(\frac{\left|x-a_{j}\right|}{1+\left|x-a_{j}\right|}\right)^{\alpha_{j}} .
$$

For $1<p<q<\infty$, assume that $u, v^{-1 /(p-1)} \Pi^{p^{\prime}(n-1)} \in D^{\infty}$ and that

$$
\left(\int_{B} \Pi(x)^{n} d x\right)^{1 / n-1} u(B)^{1 / q}\left(\int_{B} v(x)^{-1 /(p-1)} \Pi(x)^{p^{\prime}(n-1)} d x\right)^{1 / p^{\prime}} \leq c
$$

for all balls $B$. Then (1.1) holds with $c$ independent of $f$.

Corollary (1.4). Let $\left\{a_{j}\right\}_{j=1}^{m}$ be distinct points in $\mathbf{R}^{n},\left\{\beta_{j}\right\}_{j=1}^{m}$ and $\gamma$ be real numbers, $\beta_{j} \geq 0$ and $\gamma \geq 0$. Let $1<p<\infty, w(x) \in A_{p}$, and define $v(x)$ by

$$
v(x)=w(x)(1+|x|)^{\gamma} \prod_{j=1}^{m}\left(\frac{\left|x-a_{j}\right|}{1+\left|x-a_{j}\right|}\right)^{\beta_{j}} .
$$

If $1<p<q<\infty$ and $u(x)$ is any doubling weight for which

$$
|B|^{1 / n} u(B)^{1 / q} \leq c v(B)^{1 / p},
$$

then (1.1) holds with $c$ independent of $f$.

As was indicated in [2], condition (1.5) is necessary for (1.1) if $u$ and $v$ are doubling weights. We also remark that (1.5) is necessary and sufficient for the boundedness of the fractional integral of order 1 ,

$$
I_{1} f(x)=\int_{\mathbf{R}^{n}} f(y) \frac{1}{|x-y|^{n-1}} d y,
$$

from the Hardy space $H_{v}^{p}$ to $H_{u}^{q}, 0<p<q<\infty$ : see [4 and7]. 
For the case $p=q$, we need a slightly stronger hypothesis. For a weight $u$, we say that " $u^{r} \in D^{\infty}$ uniformly in $r$ near 1 " if $u^{r} \in D^{\infty}$ with doubling constant independent of $r$ for $1 \leq r<r_{0}$ for some $r_{0}>1$, i.e., if

$$
\int_{2 B} u^{r} d x \leq c \int_{B} u^{r} d x
$$

with $c$ independent of $B$ and $r, 1 \leq r<r_{0}$.

Theorem (1.6). Let $\Pi(x)$ be as in Theorem (1.2). For $1<p<\infty$, assume that $u^{r},\left\{v^{-1 /(p-1)} \Pi^{p^{\prime}(n-1)}\right\}^{r} \in D^{\infty}$ uniformly in $r$ near 1 and that

$$
\begin{aligned}
&\left(\int_{B} \Pi^{n} d x\right)^{1 / n-1} {\left[\left(\int_{B} u^{r} d x\right)^{1 / r}|B|^{1 / r^{\prime}}\right]^{1 / p} } \\
& \cdot\left[\left(\int_{B}\left\{v^{-1 /(p-1)} \Pi^{p^{\prime}(n-1)}\right\}^{r} d x\right)^{1 / r}|B|^{1 / r^{\prime}}\right]^{1 / p^{\prime}} \leq c
\end{aligned}
$$

for some $r>1$ and all balls $B$. Then (1.1) holds with $p=q$ and $c$ independent of $f$.

Corollary (1.8). Let $v(x)$ be as in Corollary (1.4). For $1<p<\infty$, assume that $u^{r} \in D^{\infty}$ uniformly in $r$ near 1 and that

$$
|B|^{1 / n}\left[\left(\int_{B} u^{r} d x\right)^{1 / r}|B|^{1 / r^{\prime}}\right]^{1 / p} \leq c v(B)^{1 / p}
$$

for some $r>1$ and all balls $B$. Then (1.1) holds with $p=q$ and $c$ independent of $f$.

In $\S 2$ we give the main lemmas which will be used to prove the results. The proofs of (1.2) and (1.4) are given in §3. The proofs of (1.6) and (1.8) are similar to these, and the main differences are discussed in $\S 4$, together with remarks about a special case when the uniform doubling conditions can be dropped. In addition to the notation used above, we shall write

$$
L_{v}^{p}=\left\{f:\|f\|_{L_{v}^{p}}=\left(\int_{\mathbf{R}^{n}}|f(x)|^{p} v(x) d x\right)^{1 / p}<\infty\right\},
$$

$1<p<\infty$. We often use the same letter $c$ to denote different constants at different occurrences.

\section{Basic Lemmas}

We begin by recalling that a one-to-one transformation $y=\varphi(x)$ of $\mathbf{R}^{n}$ is called quasi-conformal if the components $\varphi_{i}$ of $\varphi$ have first-order distributional derivatives in $L_{\text {loc }}^{n}\left(\mathbf{R}^{n}\right)$ and

$$
\left(\sum_{i, j}\left(\frac{\partial}{\partial x_{j}} \varphi_{i}(x)\right)^{2}\right)^{1 / 2} \leq c\left|J_{\varphi}(x)\right|^{1 / n} \text { a.e. }
$$


where $J_{\varphi}=\operatorname{det}\left(\partial \varphi_{i} / \partial x_{j}\right)_{i, j}$. We shall write $\varphi^{\prime}=\left(\partial \varphi_{i} / \partial x_{j}\right)_{i, j}$, and we denote the transpose of $\varphi^{\prime}$ by $\varphi^{\prime t}$.

The fact that quasi-conformal mappings are relevant to (1.1) is indicated in [3]. For the most part, we shall deal only with the special quasi-conformal maps $\varphi_{\alpha}(x)=|x|^{\alpha} x, \alpha>-1$. It is not difficult to see that

$$
\varphi_{\alpha}^{\prime}(x)=|x|^{\alpha}\left(\delta_{i j}+\alpha x_{i} x_{j}|x|^{-2}\right)
$$

and that $\left|J_{\varphi_{\alpha}}(x)\right| \approx|x|^{\alpha n}$. Moreover, the inverse $\varphi_{\alpha}^{-1}$ of $\varphi_{\alpha}$ is given by $\varphi_{\alpha}^{-1}(y)=y|y|^{-\alpha /(\alpha+1)}$ and is also quasi-conformal. A fact we shall use about $\varphi_{\alpha}^{-1}$ is that there is a positive constant $c$ depending on $\alpha$ so that if $B$ is a ball and $\varphi_{\alpha}^{-1}(B)$ denotes the image of $B$ under $\varphi_{\alpha}^{-1}$, then there exist balls $B_{1}$ and $B_{2}$ with $B_{1} \subset \varphi_{\alpha}^{-1}(B) \subset B_{2}$ and $\left|B_{2}\right| \leq c\left|B_{1}\right|$. This fact is actually true for any quasi-conformal transformation, not just for $\varphi_{\alpha}^{-1}$; see, e.g., [8]. Moreover, note that $\left|\varphi_{\alpha}^{-1}(2 B)\right| \leq c\left|\varphi_{\alpha}^{-1}(B)\right|$ since

$$
\left|\varphi_{\alpha}^{-1}(B)\right|=\int_{\varphi_{\alpha}^{-1}(B)} d x=\int_{B}\left|J_{\varphi_{\alpha}^{-1}}(y)\right| d y \approx \int_{B}|y|^{-\alpha n /(\alpha+1)} d y,
$$

and $|y|^{-\alpha n /(\alpha+1)} \in D^{\infty}$ since $-\alpha n /(\alpha+1)>-n$. By [5], a similar fact holds for any quasi-conformal map.

Lemma (2.1). Let $0<p, q<\infty$ and suppose that $f, \tilde{u}$ and $\tilde{v}$ are functions satisfying

$$
\left(\int_{\mathbf{R}^{n}}|f(y)|^{q} \tilde{u}(y) d y\right)^{1 / q} \leq A\left(\int_{\mathbf{R}^{n}}|\nabla f(y)|^{p} \tilde{v}(y) d y\right)^{1 / p}
$$

for a certain constant $A$. If $\varphi(x)$ is a quasi-conformal transformation of $\mathbf{R}^{n}$, $h=f \circ \varphi, u=(\tilde{u} \circ \varphi)\left|J_{\varphi}\right|$ and $v=(\tilde{v} \circ \varphi)\left|J_{\varphi}\right|^{1-(p / n)}$, then there is a constant $c$ depending only on $\varphi$ such that

$$
\left(\int_{\mathbf{R}^{n}}|h(x)|^{q} u(x) d x\right)^{1 / q} \leq c A\left(\int_{\mathbf{R}^{n}}|\nabla h(x)|^{p} v(x) d x\right)^{1 / p} .
$$

Proof. Let $y=\varphi(x)$ in the inequality in the hypothesis to obtain

$$
\begin{aligned}
& \left(\int_{\mathbf{R}^{n}}|(f \circ \varphi)(x)|^{q}(\tilde{\mathcal{u}} \circ \varphi)(x)\left|J_{\varphi}(x)\right| d x\right)^{1 / q} \\
& \quad \leq A\left(\int_{\mathbf{R}^{n}}|(\nabla f)(\varphi(x))|^{p}(\tilde{v} \circ \varphi)(x)\left|J_{\varphi}(x)\right| d x\right)^{1 / p} .
\end{aligned}
$$

Since

$$
\nabla(f \circ \varphi)(x)=\varphi^{\prime t}(x)[(\nabla f)(\varphi(x))] \quad \text { (matrix action) }
$$

we have

$$
(\nabla f)(\varphi(x))=\left(\varphi^{\prime t}(x)\right)^{-1}[\nabla(f \circ \varphi)(x)] \quad \text { (matrix action) }
$$


Thus, since $\left\|\varphi^{\prime-1}\right\| \leq\left\|\varphi^{\prime}\right\|^{n-1} /\left|J_{\varphi}\right| \leq c^{n-1} /\left|J_{\varphi}\right|^{1 / n}$ if $\varphi$ is quasi-conformal, we obtain

$$
|(\nabla f)(\varphi(x))| \leq c\left|J_{\varphi}(x)\right|^{-1 / n}|\nabla(f \circ \varphi)(x)|
$$

with $c$ depending on $\varphi$. The lemma now follows immediately by combining inequalities.

Lemma (2.2). Let $w(x) \in D^{\infty}$ and $\beta>0$. Let $B$ be a ball whose radius is $r$ and whose distance to the origin is $d$. Then

$$
\int_{B} w(x)(1+|x|)^{\beta} d x \approx(1+r+d)^{\beta} \int_{B} w(x) d x,
$$

and the constants in the equivalence are independent of $B, r$ and $d$.

Proof. Since $w(x) \in D^{\infty}$ and $\beta>0$, it follows from Lemma (6.3) of [7] that $w(x)(1+|x|)^{\beta} \in D^{\infty}$. In case $d \geq 2 r$, we have $|x| \approx d$ for all $x \in B$, and therefore,

$$
\int_{B} w(x)(1+|x|)^{\beta} d x \approx(1+d)^{\beta} \int_{B} w(x) d x .
$$

Since $1+d \approx 1+r+d$ when $d \geq 2 r$, the lemma follows in this case. If $d<2 r$, then $B \subset B_{4 r}(0)$ and these balls have comparable sizes. Thus, using doubling,

$$
\begin{aligned}
\int_{B} w(x)(1+|x|)^{\beta} d x & \approx \int_{|x|<4 r} w(x)(1+|x|)^{\beta} d x \\
& \approx \int_{2 r<|x|<4 r} w(x)(1+|x|)^{\beta} d x \\
& \approx(1+r)^{\beta} \int_{2 r<|x|<4 r} w(x) d x \\
& \approx(1+r)^{\beta} \int_{B} w(x) d x .
\end{aligned}
$$

Since $1+r \approx 1+r+d$ when $d<2 r$, the lemma follows.

Lemma (2.3). Let $\left\{a_{j}\right\}_{j=1}^{m}$ be distinct points in $\mathbf{R}^{n}$. Then every ball $B$ contains a ball $B^{\prime}$ with $\left|B^{\prime}\right| \approx|B|$ such that $\left|x-a_{j}\right|$ is essentially constant on $B^{\prime}$ for each $j$; i.e., there exists positive constants $c_{1}, c_{2}, c_{3}$ independent of $B, B^{\prime}$ such that $\left|B^{\prime}\right| \leq|B| \leq c_{1}\left|B^{\prime}\right|$ and $c_{2} \leq\left|x-a_{j}\right| /\left|y-a_{j}\right| \leq c_{3}$ if $x, y \in B^{\prime}$.

This fact is contained in the proof of Lemma (6.6) of [7].

We shall use Lemma (2.3) to show that if $w(x)$ and

$$
w(x) \prod_{j=1}^{m}\left(\frac{\left|x-a_{j}\right|}{1+\left|x-a_{j}\right|}\right)^{\alpha_{j}}
$$

are doubling weights and $B$ and $B^{\prime}$ are as in the lemma with $\left|x-a_{j}\right| \approx d_{j}$ for $x \in B^{\prime}$, then

$$
\int_{B} w(x) \prod_{j=1}^{m}\left(\frac{\left|x-a_{j}\right|}{1+\left|x-a_{j}\right|}\right)^{\alpha_{j}} d x \approx\left(\int_{B} w(x) d x\right) \prod_{j=1}^{m}\left(\frac{d_{j}}{1+d_{j}}\right)^{\alpha_{j}} .
$$


In fact, this follows easily by observing that due to doubling, both integrals in (2.4) are equivalent to their analogues with integration extended only over $B^{\prime}$.

Lemma (2.5). Let $\left\{a_{j}\right\}_{j=1}^{k}$ be distinct points in $\mathbf{R}^{n},\left\{\alpha_{j}\right\}_{j=1}^{k}$ and $\delta$ be real numbers, $\alpha_{j}>-1, \delta>-1$, and

$$
\prod(x)=(1+|x|)^{\delta} \prod_{j=1}^{k}\left(\frac{\left|x-a_{j}\right|}{1+\left|x-a_{j}\right|}\right)^{\alpha_{j}}
$$

Assume that $u$ and $v$ are weight functions with

$$
\begin{gathered}
\left(\int_{B} \Pi(x)^{n} d x\right)^{1 / n-1} u(B)^{1 / q}\left(\int_{B} v(x)^{-1 /(p-1)} \Pi(x)^{p^{\prime}(n-1)} d x\right)^{1 / p^{\prime}} \leq c \\
u \in D^{\infty}, \quad v^{-1 /(p-1)} \Pi^{p^{\prime}(n-1)} \in D^{\infty}
\end{gathered}
$$

for given $p$ and $q, 1<p<\infty$. Define $\tilde{u}, \tilde{v}$ and $\tilde{\Pi}$ by

$$
\begin{aligned}
& u\left(x+a_{k}\right)=\left(\tilde{u} \circ \varphi_{\alpha_{k}}\right)(x)\left|J_{\varphi_{\alpha_{k}}}(x)\right|, \\
& v\left(x+a_{k}\right)=\left(\tilde{v} \circ \varphi_{\alpha_{k}}\right)(x)\left|J_{\varphi_{\alpha_{k}}}(x)\right|^{1-(p / n),} \\
& \widetilde{\Pi}(x)=(1+|x|)^{\theta} \prod_{j=1}^{k-1}\left(\frac{\left|x-b_{j}\right|}{1+\left|x-b_{j}\right|}\right)^{\alpha_{j}},
\end{aligned}
$$

where $\theta$ satisfies $\theta\left(\alpha_{k}+1\right)+\alpha_{k} \geq \delta$ and $\left\{b_{j}\right\}_{j=1}^{k-1}$ are defined by $b_{j}=$ $\varphi_{\alpha_{k}}\left(a_{j}-a_{k}\right), j=1, \ldots, k-1$; in case $k=1$, there are no $b_{j}$ 's and we take $\widetilde{\Pi}(x)=(1+|x|)^{\theta}$. Then

(i) $\quad\left(\int_{B} \widetilde{\Pi}(x)^{n} d x\right)^{1 / n-1} \tilde{u}(B)^{1 / q}\left(\int_{B} \tilde{v}(x)^{-1 /(p-1)} \widetilde{\Pi}(x)^{p^{\prime}(n-1)} d x\right)^{1 / p^{\prime}} \leq c$,

(ii) ${ }^{\prime}$

$$
\tilde{u} \in D^{\infty}, \quad \tilde{v}^{-1 /(p-1)} \widetilde{\Pi}^{p^{\prime}(n-1)} \in D^{\infty} .
$$

Proof. First note that

$$
\Pi(x)^{n}=(1+|x|)^{\delta n} \prod_{1}^{k}\left(\frac{\left|x-a_{j}\right|}{1+\left|x-a_{j}\right|}\right)^{\alpha_{j} n} .
$$

Since $\alpha_{j} n>-n$ and $\delta n>-n$, we have $\Pi^{n} \in D^{\infty}$. Similarly, since it is easy to see that $\theta>-1$, we have $\widetilde{\Pi}^{n} \in D^{\infty}$.

Now make the change of variables $x=\varphi_{\alpha_{l}}(t)=|t|^{\alpha_{k}} t$ in (i) ${ }^{\prime}$. The product in $(i)^{\prime}$ is equivalent to

$$
\begin{aligned}
& \left(\int_{\varphi_{\alpha_{k}(B)}^{-1}} \widetilde{\Pi}\left(\varphi_{\alpha_{k}}(t)\right)^{n}|t|^{\alpha_{k} n} d t\right)^{1 / n-1}\left(\int_{\varphi_{\alpha_{k}(B)}^{-1}} u\left(t+a_{k}\right) d t\right)^{1 / q} \\
& \cdot\left(\int_{\varphi_{\alpha_{k}}^{-1}(B)}\left\{v\left(t+a_{k}\right)|t|^{-\alpha_{k} n(1-p / n)}\right\}^{-1 /(p-1)} \widetilde{\Pi}\left(\varphi_{\alpha_{k}}(t)\right)^{p^{\prime}(n-1)}|t|^{\alpha_{k} n} d t\right)^{1 / p^{\prime}}
\end{aligned}
$$


Let $\bar{a}_{j}=a_{j}-a_{k}$ for $j=1, \ldots, k-1$, so that $b_{j}=\varphi_{\alpha_{k}}\left(\bar{a}_{j}\right)$. Then since $\left|\varphi_{\alpha_{k}}(t)\right|=|t|^{\alpha_{k}+1}$,

$$
\widetilde{\Pi}\left(\varphi_{\alpha_{k}}(t)\right) \approx\left(1+|t|^{\alpha_{k}+1}\right)^{\theta} \prod_{j=1}^{k-1}\left(\frac{\left|\varphi_{\alpha_{k}}(t)-\varphi_{\alpha_{k}}\left(\bar{a}_{j}\right)\right|}{1+\left|\varphi_{\alpha_{k}}(t)-\varphi_{\alpha_{k}}\left(\bar{a}_{j}\right)\right|}\right)^{\alpha_{j}}
$$

But

$$
\frac{\left|\varphi_{\alpha_{k}}(t)-\varphi_{\alpha_{k}}\left(\bar{a}_{j}\right)\right|}{1+\left|\varphi_{\alpha_{k}}(t)-\varphi_{\alpha_{k}}\left(\bar{a}_{j}\right)\right|} \approx \frac{\left|t-\bar{a}_{j}\right|(1+|t|)^{\alpha_{k}}}{1+\left|t-\bar{a}_{j}\right|(1+|t|)^{\alpha_{k}}} \approx \frac{\left|t-\bar{a}_{j}\right|}{1+\left|t-\bar{a}_{j}\right|} .
$$

Thus, the integrands in the first and third terms of (2.6) are equivalent to the following two expressions, respectively:

$$
\left\{(1+|t|)^{\theta\left(\alpha_{k}+1\right)+\alpha_{k}} \prod_{j=1}^{k-1}\left(\frac{\left|t-\bar{a}_{j}\right|}{1+\left|t-\bar{a}_{j}\right|}\right)^{\alpha_{j}} \cdot\left(\frac{|t|}{1+|t|}\right)^{\alpha_{k}}\right\}^{n}
$$

and

$v\left(t+a_{k}\right)^{-1 /(p-1)}\left\{(1+|t|)^{\theta\left(\alpha_{k}+1\right)+\alpha_{k}} \prod_{j=1}^{k-1}\left(\frac{\left|t-\bar{a}_{j}\right|}{1+\left|t-\bar{a}_{j}\right|}\right)^{\alpha_{j}} \cdot\left(\frac{|t|}{1+|t|}\right)^{\alpha_{k}}\right\}^{p^{\prime}(n-1)}$.

Recalling the definition of $\Pi$, we see that these are in turn equivalent respectively to

$$
\left\{(1+|t|)^{\theta\left(\alpha_{k}+1\right)+\alpha_{k}-\delta} \Pi\left(t+a_{k}\right)\right\}^{n}
$$

and

$$
v\left(t+a_{k}\right)^{-1 /(p-1)}\left\{(1+|t|)^{\theta\left(\alpha_{k}+1\right)+\alpha_{k}-\delta} \Pi\left(t+a_{k}\right)\right\}^{p^{\prime}(n-1)} .
$$

Thus, replacing $t$ by $\tau_{-a_{k}}(t)=t-a_{k}$ in each integral in (2.6), we see (2.6) is equivalent to

$$
\begin{gathered}
\left(\int_{\tau_{a_{k}}\left(\varphi_{u_{k}}^{-1}(B)\right)}\left\{(1+|t|)^{\theta\left(\alpha_{k}+1\right)+\alpha_{k}-\delta} \Pi(t)\right\}^{n} d t\right)^{1 / n-1} u\left(\tau_{a_{k}}\left(\varphi_{\alpha_{k}}^{-1}(B)\right)\right)^{1 / q} \\
\cdot\left(\int_{\tau_{a_{k}\left(\varphi_{i_{k}}(B)\right)}} v(t)^{-1 /(p-1)}\left\{(1+|t|)^{\theta\left(\alpha_{k}+1\right)+\alpha_{k}-\delta} \Pi(t)\right\}^{p^{\prime}(n-1)} d t\right)^{1 / p^{\prime}} .
\end{gathered}
$$

We note that all the integrands in (2.7) are doubling: this follows from Lemma (6.3) of [7], the fact that $\theta\left(\alpha_{k}+1\right)+\alpha_{k}-\delta \geq 0$, hypothesis (ii), and the fact that $\Pi^{n} \in D^{\infty}$. Hence, since $\varphi_{\alpha_{k}}^{-1}$ is quasi-conformal, if we replace the set $\tau_{a_{k}}\left(\varphi_{\alpha_{k}}^{-1}(B)\right)$ in (2.7) by the smallest ball containing this set, we obtain an expression equivalent to (2.7). Let us denote this ball by $B$ again. Now using Lemma (2.2) and the relation of the exponents in (2.7) it follows that the factor $(1+|t|)^{\theta\left(\alpha_{k}+1\right)+\alpha_{k}-\delta}$ can be cancelled out of both integrands in (2.7). Thus, (2.7) is bounded due to hypothesis (i). This proves (i) '. 
Finally, (ii) ${ }^{\prime}$ was essentially proved in the course of proving (i) ${ }^{\prime}$. For example, to show that $\tilde{u}(2 B) \leq c \tilde{u}(B)$, recall first from above that

$$
\tilde{u}(B)=u\left(\tau_{a_{k}}\left(\varphi_{\alpha_{k}}^{-1}(B)\right)\right) .
$$

Since $\varphi_{\alpha_{k}}^{-1}$ is quasi-conformal, the smallest ball containing $\varphi_{\alpha_{k}}^{-1}(B)$ has volume comparable to that of the smallest ball containing $\varphi_{\alpha_{k}}^{-1}(2 B)$. Since $u \in D^{\infty}$, it then follows easily that $\tilde{u} \in D^{\infty}$. The argument showing that $\tilde{v}^{-1 /(p-1)} \tilde{\Pi}^{p^{\prime}(n-1)}$ $\in D^{\infty}$ is similar. This completes the proof of Lemma (2.5).

Now let $f$ be a function which satisfies

$$
|f(x)| \leq c_{0} I_{1}(|\nabla f|)(x)
$$

for a.e. $x$, where $c_{0}$ is a constant independent of $x$ and $f$, and $I_{1}$ is the fractional integral defined in $\S 1$. Any $f \in C_{0}^{\infty}\left(\mathbf{R}^{n}\right)$ satisfies (2.8); more generally, any $f$ with compact support which satisfies

$$
|f(x)-f(y)| \leq c_{0}^{\prime}|x-y| \int_{0}^{1}|(\nabla f)(y+t(x-y))| d t, \quad x, y \in \mathbf{R}^{n},
$$

also satisfies (2.8), as can easily be seen by integrating (2.9) with respect to $y$ over the surface of a large sphere centered at $x$. Another way to see that (2.9) implies (2.8) is by examining the proofs in [3].

It follows from the result of [6] for $I_{1}$ that if $u$ and $v^{-1 /(p-1)}$ are doubling weights with

$$
|B|^{1 / n-1} u(B)^{1 / q}\left(\int_{B} v(x)^{-1 /(p-1)} d x\right)^{1 / p^{\prime}} \leq c,
$$

$1<p<q<\infty$, and if $f$ satisfies (2.8) then

$$
\left(\int|f(x)|^{q} u(x) d x\right)^{1 / q} \leq c\left(\int|\nabla f(x)|^{p} v(x) d x\right)^{1 / p}
$$

with $c$ independent of $f$.

We now introduce some useful notation. Given distinct points $\left\{a_{j}\right\}_{j=1}^{m}$ in $\mathbf{R}^{n}$ and a sequence $\left\{\alpha_{j}\right\}_{j=1}^{m}$ in $\mathbf{R}^{1}$, define sequences $\left\{a_{j}^{(k)}\right\}_{j=1}^{k}, k=1, \ldots, m$, as follows. For $k=m$, let $a_{j}^{(m)}=a_{j}, j=1, \ldots, m$, and for $k=m-1$, $m-2, \ldots, 1$, define $a_{j}^{(k)}$ successively by

$$
a_{j}^{(k)}=\varphi_{\alpha_{k+1}}\left(a_{j}^{(k+1)}-a_{k+1}^{(k+1)}\right), \quad j=1, \ldots, k .
$$

For a given $k$, the $a_{j}^{(k)}$ are distinct and different from 0 . We will use the one-to-one transformations $T_{k}$ defined by

$$
T_{k}(x)=\varphi_{\alpha_{k}}\left(x-a_{k}^{(k)}\right), \quad k=1, \ldots, m, x \in \mathbf{R}^{n} .
$$

Each $T_{k}$ is a translation followed by a quasi-conformal mapping. Note that

$$
T_{k}^{-1}(x)=\varphi_{\alpha_{k}}^{-1}(x)+a_{k}^{(k)} \text {. }
$$


Lemma (2.13). Let $\left\{a_{j}\right\}_{j=1}^{m}$ be distinct points in $\mathbf{R}^{n},\left\{\alpha_{j}\right\}_{j=1}^{m}$ satisfy $\alpha_{j}>-1$, $\delta>-1$, and $\prod_{j=1}^{m}\left(\alpha_{j}+1\right) \geq \delta+1$. Let

$$
\Pi(x)=(1+|x|)^{\delta} \prod_{j=1}^{m}\left(\frac{\left|x-a_{j}\right|}{1+\left|x-a_{j}\right|}\right)^{\alpha_{j}},
$$

and for $1<p<q<\infty$, assume that

$$
\left(\int_{B} \Pi(x)^{n} d x\right)^{1 / n-1} u(B)^{1 / q}\left(\int_{B} v(x)^{-1 /(p-1)} \Pi(x)^{p^{\prime}(n-1)} d x\right)^{1 / p^{\prime}} \leq c
$$

and that $u, v^{-1 /(p-1)} \Pi^{p^{\prime}(n-1)} \in D^{\infty}$. Then

$$
\left(\int|g(x)|^{q} u(x) d x\right)^{1 / q} \leq c\left(\int|\nabla g(x)|^{p} v(x) d x\right)^{1 / p}
$$

for every $g$ of the form $g=f \circ T_{1} \circ \cdots \circ T_{m}$ where $f$ satisfies (2.8) and $\left\{T_{k}\right\}$ are defined by (2.11).

Proof. The proof is by induction on $m$. If $m=1$, we have $\alpha_{1} \geq \delta$, and we may apply Lemma (2.5) with $k=1$ and $\theta=0$ to obtain the representations

$$
\begin{aligned}
& u\left(x+a_{1}\right)=\left(\tilde{u} \circ \varphi_{\alpha_{1}}\right)(x)\left|J_{\varphi_{\alpha_{1}}}(x)\right|, \\
& v\left(x+a_{1}\right)=\left(\tilde{v} \circ \varphi_{\alpha_{1}}\right)(x)\left|J_{\varphi_{\alpha_{1}}}(x)\right|^{1-(p / n)},
\end{aligned}
$$

where

$$
|B|^{1 / n-1} \tilde{u}(B)^{1 / q}\left(\int_{B} \tilde{v}^{-1 /(p-1)} d x\right)^{1 / p^{\prime}} \leq c
$$

and $\tilde{u}, \tilde{v}^{-1 /(p-1)} \in D^{\infty}$ (cf. (i) ' and (ii)' of Lemma (2.5)). By the result of [6] mentioned earlier, we have $\|f\|_{L_{u}^{q}} \leq c\|\nabla f\|_{L_{v}^{p}}$ for any $f$ which satisfies (2.8). Therefore, by Lemma (2.1),

$$
\left(\int|h(x)|^{q} u\left(x+a_{1}\right) d x\right)^{1 / q} \leq c\left(\int|\nabla h(x)|^{p} v\left(x+a_{1}\right) d x\right)^{1 / p}
$$

if $h$ has the form $h=f \circ \varphi_{\alpha_{1}}$ for such $f$. Replacing $x$ by $x-a_{1}$ and noting that $h\left(x-a_{1}\right)=\left(f \circ T_{1}\right)(x)$, we obtain the desired inequality

$$
\left(\int|g(x)|^{q} u(x) d x\right)^{1 / q} \leq c\left(\int|\nabla g(x)|^{p} v(x) d x\right)^{1 / p}
$$

if $g=f \circ T_{1}$ with $f$ satisfying (2.8).

Assuming next that the lemma is true for $m-1$, we will prove it for $m$. By hypothesis, $\prod_{j=1}^{m}\left(\alpha_{j}+1\right) \geq \delta+1$. Thus, letting $\theta$ be defined by $\theta+1=$ $\prod_{j=1}^{m-1}\left(\alpha_{j}+1\right)$, we have $\theta\left(\alpha_{m}+1\right)+\alpha_{m} \geq \delta$. We may then apply Lemma (2.5) with this value of $\theta$ and with $k=m$ to obtain

$$
\left(\int_{B} \tilde{\Pi}(x)^{n} d x\right)^{1 / n-1} \tilde{u}(B)^{1 / q}\left(\int_{B} \tilde{v}(x)^{-1 /(p-1)} \tilde{\Pi}(x)^{p^{\prime}(n-1)} d x\right)^{1 / p^{\prime}} \leq c
$$


and $\tilde{u} \in D^{\infty}$ and $\tilde{v}^{-1 /(p-1)} \widetilde{\Pi}^{p^{\prime}(n-1)} \in D^{\infty}$, where

$$
\begin{aligned}
& u\left(x+a_{m}\right)=\left(\tilde{u} \circ \varphi_{\alpha_{m}}\right)(x)\left|J_{\varphi_{r_{m}}}(x)\right|, \\
& v\left(x+a_{m}\right)=\left(\tilde{v} \circ \varphi_{\alpha_{m}}\right)(x)\left|J_{\varphi_{r_{m}}}(x)\right|^{1-(p / n)}, \\
& \widetilde{\Pi}(x)=(1+|x|)^{\theta} \prod_{j=1}^{m-1}\left(\frac{\left|x-a_{j}^{(m-1)}\right|}{1+\left|x-a_{j}^{(m-1)}\right|}\right)^{\alpha_{j}} .
\end{aligned}
$$

Clearly, $\theta>-1$, and by definition, $\prod_{1}^{m-1}\left(\alpha_{j}+1\right)=\theta+1$. Thus, by the induction hypothesis, we have $\|g\|_{L_{i}^{q}} \leq c\|\nabla g\|_{L_{i}^{p}}$ for any $g$ of the form $g=$ $f \circ \tilde{T}_{1} \circ \cdots \circ \tilde{T}_{m-1}$ where $f$ satisfies $(2.8)$ and $\left\{\tilde{T}_{k}\right\}_{1}^{m-1}$ are defined as usual starting with the points $\left\{a_{j}^{(m-1)}\right\}_{j=1}^{m-1}$ and the exponents $\left\{\alpha_{j}\right\}_{j=1}^{m-1}$. It is easy to see that $\widetilde{T}_{k}=T_{k}$. Now applying Lemma (2.1), we obtain

$$
\left(\int|h(x)|^{q} u\left(x+a_{m}\right) d x\right)^{1 / q} \leq c\left(\int|\nabla h(x)|^{p} v\left(x+a_{m}\right) d x\right)^{1 / p}
$$

if $h=\left(f \circ T_{1} \circ \cdots \circ T_{m-1}\right) \circ \varphi_{\alpha_{m}}$. Since $h\left(x-a_{m}\right)=\left(f \circ T_{1} \circ \cdots \circ T_{m}\right)(x)$, the desired result follows by replacing $x$ by $x-a_{m}$ in the integrals. This completes the proof of Lemma (2.13).

Lemma (2.14). Let $\left\{a_{j}\right\}_{1}^{m}$ be distinct points in $\mathbf{R}^{n},\left\{\alpha_{j}\right\}_{1}^{m}$ satisfy $\alpha_{j}>-1$, and define $\left\{T_{k}\right\}_{1}^{m}$ by (2.11). If $g \in C_{0}^{\infty}$ then the function $f=g \circ T_{m}^{-1} \circ \cdots \circ T_{1}^{-1}$ satisfies (2.8). In fact, $f$ has compact support and satisfies (2.9). In particular, any $g \in C_{0}^{\infty}$ may be written $g=f \circ T_{1} \circ \cdots \circ T_{m}$ where $f$ satisfies (2.8).

Proof. We shall first show that

(i) $T_{k+1} a_{j}^{(k+1)}=a_{j}^{(k)}, k \geq j$,

(ii) $T_{k} \cdots T_{m} a_{k}^{(m)}=0, k \leq m$,

where $a_{j}^{(k)}$ are defined by (2.10) and $T_{k} \cdots T_{m}=T_{k} \circ \cdots \circ T_{m}$.

In fact, (i) is immediate from (2.11) and (2.10). For (ii), we have by (i) that

$$
T_{k} \cdots T_{m} a_{k}^{(m)}=T_{k} \cdots T_{m-1} a_{k}^{(m-1)}=\cdots=T_{k} a_{k}^{(k)}=0 \quad \text { by }(2.11) .
$$

To complete the proof of Lemma (2.14), let $g \in C_{0}^{\infty}$ and $f=g \circ T_{m}^{-1} \cdots T_{1}^{-1}$. Thus, $f \circ T_{1} \cdots T_{m}=g$. By repeated use of the formula $\nabla(G \circ S)(y)=$ $S^{\prime t}(y) \nabla G(y)$, where $G$ is real-valued and $S$ is a differentiable transformation of $\mathbf{R}^{n}$, we have

$$
\begin{aligned}
\nabla f(x)=\left.\left.\left.\left(T_{1}^{-1}\right)^{\prime t}\right|_{x}\left(T_{2}^{-1}\right)^{\prime t}\right|_{T_{1}^{-1} x}\left(T_{3}^{-1}\right)^{\prime t}\right|_{T_{2}^{-1} T_{1}^{-1} x} \\
\left.\left.\cdots\left(T_{m}^{-1}\right)^{\prime t}\right|_{T_{m-1}^{-1} \cdots T_{1}^{-1} x}(\nabla g)\right|_{T_{m}^{-1} \cdots T_{1}^{-1} x} .
\end{aligned}
$$

Note that $\left(T_{j}^{-1}\right)^{\prime}=\left(\varphi_{\alpha_{j}}^{-1}\right)^{\prime}$, and that this can be singular only at 0 . Thus, $|\nabla f(x)|$ can be singular only at the points $x_{1}, \ldots, x_{m}$ defined by $x_{1}=0$, $T_{1}^{-1} x_{2}=0, T_{2}^{-1} T_{1}^{-1} x_{3}=0, \ldots, T_{m-1}^{-1} \cdots T_{1}^{-1} x_{m}=0$. Thus, $x_{1}=0, x_{2}=$ 
$T_{1} 0, x_{3}=T_{1} T_{2} 0, \ldots, x_{m}=T_{1} \cdots T_{m-1} 0$. Combining this with (ii) above, we have

$$
\begin{aligned}
& x_{1}=0=T_{1} \cdots T_{m} a_{1}^{(m)} \\
& x_{2}=T_{1} 0=T_{1} \cdots T_{m} a_{2}^{(m)} \\
& \vdots \\
& x_{m}=T_{1} \cdots T_{m-1} 0=T_{1} \cdots T_{m} a_{m}^{(m)} .
\end{aligned}
$$

Since the transformation $T_{1} \cdots T_{m}$ is one-to-one and the points $a_{1}^{(m)}, \ldots, a_{m}^{(m)}$ $\left(=a_{1}, \ldots, a_{m}\right.$, respectively $)$ are distinct, we see that $x_{1}, \ldots, x_{m}$ are distinct.

We have shown that the singularities of $\nabla f$ are at most a finite number of distinct points $x_{1}, \ldots, x_{m}$. The norm (as a transformation) of $\left(\varphi_{\alpha_{j}}^{-1}\right)^{\prime}$ is equivalent to $\left|J_{\varphi_{a_{j}}^{-1}}\right|^{1 / n}$ since $\varphi_{\alpha_{j}}^{-1}$ is quasi-conformal. Since

$$
\left|J_{\varphi_{\alpha_{j}}^{-1}}(x)\right| \approx|x|^{-\alpha_{j} n /\left(\alpha_{j}+1\right)},
$$

it follows from (2.15) that

$$
|\nabla f(x)| \leq c_{g} \prod_{j=1}^{m}\left|x-x_{j}\right|^{-\alpha_{j} /\left(\alpha_{j}+1\right)} .
$$

Since $\alpha_{j} /\left(\alpha_{j}+1\right)<1$, we see that $f$ is absolutely continuous on lines and that (2.9) holds. This completes the proof of Lemma (2.14).

\section{Proof of Theorem (1.2)}

If we assume that $\Pi\left(\alpha_{j}+1\right) \geq \delta+1$, Theorem (1.2) follows immediately by combining Lemmas (2.13) and (2.14). If $\Pi\left(\alpha_{j}+1\right)<\delta+1$, pick $\bar{\alpha}_{j}$ satisfying $\bar{\alpha}_{j} \geq \alpha_{j}$ and $\Pi\left(\bar{\alpha}_{j}+1\right) \geq \delta+1$. Let

$$
\bar{\Pi}(x)=(1+|x|)^{\delta} \prod_{j=1}^{m}\left(\frac{\left|x-a_{j}\right|}{1+\left|x-a_{j}\right|}\right)^{\bar{\alpha}_{j}} .
$$

Thus,

$$
\bar{\Pi}(x)=\Pi(x) \prod_{j=1}^{m}\left(\frac{\left|x-a_{j}\right|}{1+\left|x-a_{j}\right|}\right)^{\varepsilon_{j}} \quad \text { where } \varepsilon_{j}=\bar{\alpha}_{j}-\alpha_{j} \geq 0 .
$$

It follows that $\bar{\Pi} \in D^{\infty}$ and also, since $v^{-1 /(p-1)} \Pi^{p^{\prime}(n-1)} \in D^{\infty}$ and $p^{\prime}(n-1)>$ 0 , that $v^{-1 /(p-1)} \bar{\Pi}^{p^{\prime}(n-1)} \in D^{\infty}$. Moreover, by Lemma (2.3), given a ball $B$, there is a ball $B^{\prime} \subset B$ with $\left|B^{\prime}\right| \approx|B|$ such that each $\left|x-a_{j}\right|$ is essentially 
constant on $B^{\prime}$. If $\left|x-a_{j}\right| \approx d_{j}$ for $x \in B^{\prime}$, we then have (cf. (2.4))

$$
\begin{aligned}
& \left(\int_{B} v^{-1 /(p-1)} \bar{\Pi}^{p^{\prime}(n-1)} d x\right)^{1 / p^{\prime}} \\
& \quad \approx \prod_{j=1}^{m}\left(\frac{d_{j}}{1+d_{j}}\right)^{\varepsilon_{j}(n-1)}\left(\int_{B} v^{-1 /(p-1)} \Pi^{p^{\prime}(n-1)} d x\right)^{1 / p^{\prime}} .
\end{aligned}
$$

Similarly,

$$
\left(\int_{B} \bar{\Pi}^{n} d x\right)^{1 / n-1} \approx \prod_{j=1}^{m}\left(\frac{d_{j}}{1+d_{j}}\right)^{-\varepsilon_{j}(n-1)}\left(\int_{B} \Pi^{n} d x\right)^{1 / n-1} .
$$

It follows that (1.3) implies the analogue of (1.3) with $\Pi$ replaced by $\bar{\Pi}$. We thus obtain the general case of Theorem (1.2) from the case for $\bar{\Pi}$.

Proof of Corollary (1.4). Define $\Pi(x)$ as in Theorem (1.2) with $\delta=\gamma /(n-1) p$ and $\alpha_{j}=\beta_{j} / p(n-1)$. Note that the integrand of the third factor in (1.3) is then

$$
v(x)^{-1 /(p-1)} \Pi(x)^{p^{\prime}(n-1)}=w(x)^{-1 /(p-1)} .
$$

Since $w \in A_{p}$, we have $w^{-1 /(p-1)} \in A_{p^{\prime}}$; in particular, $v^{-1 /(p-1)} \Pi^{p^{\prime}(n-1)} \in D^{\infty}$. Thus, the corollary will follow from Theorem (1.2) if we verify (1.3). Since $|B|^{1 / n} u(B)^{1 / q} \leq c v(B)^{1 / p}$, it is enough to show that

$$
\left(\int_{B} \Pi^{n} d x\right)^{1 / n-1}\left(\int_{B} w^{-1 /(p-1)} d x\right)^{1 / p^{\prime}} \leq c|B|^{1 / n} / v(B)^{1 / p} .
$$

Since $\gamma, \beta_{j} \geq 0$, we see that $v \in D^{\infty}$ by [7]. Thus, by the analogue of (2.4) and the homogeneity of the powers above, (3.1) is equivalent to

$$
\left(\int_{B} 1 d x\right)^{1 / n-1}\left(\int_{B} w^{-1 /(p-1)} d x\right)^{1 / p^{\prime}} \leq c|B|^{1 / n} /\left(\int_{B} w d x\right)^{1 / p} .
$$

This inequality is true since $w \in A_{p}$.

\section{Proof OF (1.6) AND (1.8)}

The proofs of the results for $q=p$ are similar to those for $q>p$. The main changes which are necessary are alternate versions of Lemmas (2.5) and (2.13). We list these versions in the next two lemmas, and indicate their proofs below.

Lemma (4.1). Let $\Pi(x)$ be as in Lemma (2.5). Assume that $u$ and $v$ are weight functions with

$$
\begin{aligned}
&\left(\int_{B} \Pi^{n} d x\right)^{1 / n-1}\left[\left(\int_{B} u^{r} d x\right)^{1 / r}|B|^{1 / r^{\prime}}\right]^{1 / q} \\
& \cdot\left[\left(\int_{B}\left\{v^{-1 /(p-1)} \Pi^{p^{\prime}(n-1)}\right\}^{r} d x\right)^{1 / r}|B|^{1 / r^{\prime}}\right]^{1 / p^{\prime}} \leq c
\end{aligned}
$$

for some $r>1$, 


$$
u^{r},\left\{v^{-1 /(p-1)} \Pi^{p^{\prime}(n-1)}\right\}^{r} \in D^{\infty} \quad \text { uniformly in } r \text { near } 1 \text {. }
$$

Define $\tilde{u}, \tilde{v}$ and $\widetilde{\Pi}$ as in Lemma (2.5), with $\theta$ as described there. Then the analogues of (i) and (ii) for $\tilde{u}, \tilde{v}, \widetilde{\Pi}$ hold with the same $p$ and $q, 1<p<\infty$.

As an analogue of Lemma (2.13), we have

Lemma (4.2). Let $1<p<\infty$, and assume that $u, v$ and $\Pi$ satisfy (i) and (ii) of Lemma (4.1) with $q=p$, where $\Pi$ is as in Lemma (2.13). Then the conclusion of Lemma (2.13) holds with $q=p$, i.e.,

$$
\left(\int|g(x)|^{p} u(x) d x\right)^{1 / p} \leq c\left(\int|\nabla g(x)|^{p} v(x) d x\right)^{1 / p}
$$

for every $g$ of the form $g=f \circ T_{1} \circ \cdots \circ T_{m}$ where $f$ satisfies (2.8) and $\left\{T_{k}\right\}$ are defined by (2.11).

Taking these lemmas momentarily for granted, the proof of Theorem (1.6) follows from Lemmas (4.2) and (2.14) by the same method that was used to prove Theorem (1.2), using $\bar{\Pi}$ as defined there. The only new feature required is using the fact that if $w$ is a weight for which $w^{r} \in D^{\infty}$ uniformly in $r$ near 1 then given $\mu, \nu, \sigma$ and $\tau$ with $\mu, \nu \geq 0$ and $-\infty<\sigma, \tau<+\infty$, the weights

$$
w(x)^{r}\left\{(1+|x|)^{\mu}\left(\frac{|x|}{1+|x|}\right)^{\nu}\right\}^{r}\left\{(1+|x|)^{\sigma}\left(\frac{|x|}{1+|x|}\right)^{\tau}\right\}^{1-r}
$$

also belong to $D^{\infty}$ uniformly in $r$ near 1 ; this is easily proved by examining the proof of Lemma (6.3) of [7].

To deduce Corollary (1.8) from Theorem (1.6) we must check the hypothesis of Theorem (1.6) with $\Pi$ defined by

$$
\Pi(x)^{p(n-1)}=(1+|x|)^{\gamma} \prod_{j}\left(\frac{\left|x-a_{j}\right|}{1+\left|x-a_{j}\right|}\right)^{\beta_{j}} .
$$

Since by hypothesis we have

$$
v(x)=w(x)(1+|x|)^{\gamma} \prod_{j}\left(\frac{\left|x-a_{j}\right|}{1+\left|x-a_{j}\right|}\right)^{\beta_{j}},
$$

it follows that $v=w \Pi^{p(n-1)}$, or $v^{-1 /(p-1)} \Pi^{p^{\prime}(n-1)}=w^{-1 /(p-1)}$. Since $w \in A_{p}$, we have $w^{-1 /(p-1)} \in A_{p^{\prime}}$, and therefore $w^{-r_{0} /(p-1)} \in A_{p^{\prime}}$ for some $r_{0}>1$. It then follows easily that $\left\{v^{-1 /(p-1)} \Pi^{p^{\prime}(n-1)}\right\}^{r}=w^{-r /(p-1)} \in D^{\infty}$ uniformly in $r$ near 1. Also, by hypothesis, $u^{r} \in D^{\infty}$ uniformly in $r$ near 1 . Thus, we only need to check that (1.7) holds. The third factor in (1.7) is now

$$
\left[\left(\int_{B} w^{-r /(p-1)} d x\right)^{1 / r}|B|^{1 / r^{\prime}}\right]^{1 / p^{\prime}} \approx\left(\int_{B} w^{-1 /(p-1)} d x\right)^{1 / p^{\prime}}
$$


for $r$ near 1 since $w^{-1 /(p-1)} \in A_{p^{\prime}}$. Thus, since $w \in A_{p},(1.7)$ is the same as

$$
\left(\int_{B} \Pi^{n} d x\right)^{1 / n-1}\left[\left(\int_{B} u^{r} d x\right)^{1 / r}|B|^{1 / r^{\prime}}\right]^{1 / p} \leq c|B|^{-1}\left(\int_{B} w d x\right)^{1 / p} .
$$

However, since $v=\Pi^{p(n-1)} w$ and $\gamma, \beta_{j} \geq 0$, we see that $v \in D^{\infty}$, and the last inequality follows from (1.9) by factoring out the powers of $\Pi$ (see (2.4)).

We now turn to the proofs of Lemma (4.1) and (4.2). Lemma (4.2) follows from Lemma (4.1) in the same way that Lemma (2.13) follows from Lemma (2.5) with one change: namely, we use the result from [6] that $\|f\|_{L_{u}^{p}} \leq c\|\nabla f\|_{L_{v}^{p}}$ for any $f$ which satisfies $(2.8)$, provided that

$$
|B|^{1 / n-1}\left[\left(\int_{B} u^{r} d x\right)^{1 / r}|B|^{1 / r^{\prime}}\right]^{1 / p}\left[\left(\int_{B} v^{-r /(p-1)} d x\right)^{1 / r}|B|^{1 / r^{\prime}}\right]^{1 / p^{\prime}} \leq c
$$

for some $r>1$.

To prove Lemma (4.1), we follow the outline of the proof of Lemma (2.5). First note that condition (i) of Lemma (4.1) is nested in $r$, i.e., if it holds for $r=r_{0}$, then by Hölder's inequality it also holds for $1 \leq r<r_{0}$. Using (i) and (ii), and temporarily assuming the analogue of (ii) for $\tilde{u}, \tilde{v}, \widetilde{\Pi}$, let us show that the analogue of (i) for $\tilde{u}, \tilde{v}, \widetilde{\Pi}$ holds. Actually, we will show something which is more general: without any doubling assumptions, we will show that

$$
\begin{aligned}
& \left(\int_{B} \tilde{\Pi}^{n} d x\right)^{1 / n-1}\left[\left(\int_{B}\left\{\frac{\tilde{u}}{\widetilde{\Pi}^{n}}\right\}^{r} \widetilde{\Pi}^{n} d x\right)^{1 / r}\left(\int_{B} \tilde{\Pi}^{n} d x\right)^{1 / r^{\prime}}\right]^{1 / q} \\
& \cdot\left[\left(\int_{B}\left\{\frac{\tilde{v}^{-1 /(p-1)} \widetilde{\Pi}^{p^{\prime}(n-1)}}{\widetilde{\Pi}^{n}}\right\}^{r} \widetilde{\Pi}^{n} d x\right)^{1 / r}\left(\int_{B} \tilde{\Pi}^{n} d x\right)^{1 / r^{\prime}}\right]^{1 / p^{\prime}} \leq c
\end{aligned}
$$

if it is true that the following analogous inequality holds with $\Pi^{*}$ defined by

$$
\begin{gathered}
\Pi^{*}(x)=(1+|x|)^{\theta\left(\alpha_{k}+1\right)+\alpha_{k}-\delta} \Pi(x) \quad(\theta, \delta \text { arbitrary }): \\
\left(\int_{B} \Pi^{* n} d x\right)^{1 / n-1}\left[\left(\int_{B}\left\{\frac{u}{\Pi^{* n}}\right\} \Pi^{* n} d x\right)^{1 / r}\left(\int_{B} \Pi^{*} d x\right)^{1 / r^{\prime}}\right]^{-1 / q} \\
\cdot\left[\left(\int_{B}\left\{\frac{v^{-1 /(p-1)} \Pi^{* p^{\prime}(n-1)}}{\Pi^{* n}}\right\}^{r} \Pi^{* n} d x\right)^{1 / r}\left(\int_{B} \Pi^{* n} d x\right)^{1 / r^{\prime}}\right]^{1 / p^{\prime}} \leq c .
\end{gathered}
$$

If we assume the doubling conditions and also that $\theta\left(\alpha_{k}+1\right)+\alpha_{k}-\delta \geq 0$, this will show what we want since then (4.3) is equivalent to the analogue of (i) for $\tilde{u}, \tilde{v}, \widetilde{\Pi}$, and (4.4) is equivalent to (i) provided $r$ is near 1. This follows from (2.4) and the remark made after the statement of Lemma (4.2); also, for 
the first and third factors in (4.4), we use the fact that $\lambda=\theta\left(\alpha_{k}+1\right)+\alpha_{k}-\delta \geq 0$ together with the identities

$$
\Pi^{* n}=\Pi^{n}(1+|x|)^{\lambda n}
$$

and

$$
v^{-1 /(p-1)} \Pi^{* p^{\prime}(n-1)}=\left[v^{-1 /(p-1)} \Pi^{p^{\prime}(n-1)}\right](1+|x|)^{\lambda p^{\prime}(n-1)},
$$

respectively.

To see that (4.4) implies (4.3), let $x=\varphi_{\alpha_{k}}(t)=|t|^{\alpha_{k}} t$ in (4.3), and recall that (by the argument between (2.6). and (2.7))

$$
\begin{aligned}
\widetilde{\Pi}\left(\varphi_{\alpha_{k}}(t)\right)|t|^{\alpha_{k}} & \approx(1+|t|)^{\lambda} \Pi\left(t+a_{k}\right), \quad \lambda=\theta\left(\alpha_{k}+1\right)+\alpha_{k}-\delta, \\
& \approx \Pi^{*}\left(t+a_{k}\right)
\end{aligned}
$$

by definition of $\Pi^{*}$. Using these equivalences, the definitions of $\tilde{u}$ and $\tilde{v}$, and the fact that

$$
\alpha_{k} n\left(\frac{p}{n}-1\right)\left(\frac{-1}{p-1}\right)+\alpha_{k} n=\alpha_{k} p^{\prime}(n-1),
$$

we see that (4.3) is equivalent to the boundedness of the product of the following three terms:

$$
\begin{aligned}
& \left(\int_{\varphi_{a_{k}}^{-1}(B)} \Pi^{*}\left(t+a_{k}\right)^{n} d t\right)^{1 / n-1+1 / r^{\prime} q+1 / r^{\prime} p^{\prime}}, \\
& \left(\int_{\varphi_{a_{k}}^{-1}(B)}\left\{\frac{u\left(t+a_{k}\right)}{\Pi^{*}\left(t+a_{k}\right)^{n}}\right\}^{r} \Pi^{*}\left(t+a_{k}\right)^{n} d t\right)^{1 / r q}, \\
& \left(\int_{\varphi_{n_{k}}^{-1}(B)}\left\{\frac{v\left(t+a_{k}\right)^{-1 /(p-1)} \Pi^{*}\left(t+a_{k}\right)^{p^{\prime}(n-1)}}{\Pi^{*}\left(t+a_{k}\right)^{n}}\right\}^{r} \Pi^{*}\left(t+a_{k}\right)^{n} d t\right)^{1 / r p^{\prime}} .
\end{aligned}
$$

Now translate each integral by $-a_{k}$, and replace the translated ball $\tau_{a_{k}}\left(\varphi_{\alpha_{k}}^{-1}(B)\right)$ by the least ball containing it. The resulting product is just the expression in (4.4) for this ball, and thus (4.3) follows.

It remains only to show that (ii) implies the analogue of (ii) for $\tilde{u}, \tilde{v}, \tilde{\Pi}$. We have

$$
\begin{aligned}
\int_{B} \tilde{u}(t)^{r} d t & =\int_{B}\left\{u\left(\varphi_{\alpha_{k}}^{-1}(t)+a_{k}\right) \mid\left(\left.\varphi_{\alpha_{k}}^{-1}(t)\right|^{-\alpha_{k} n}\right\}^{r} d t\right. \\
& =\int_{\varphi_{\alpha_{k}}^{-1}(B)} u\left(x+a_{k}\right)^{r}|x|^{\alpha_{k} n(1-r)} d x
\end{aligned}
$$

Since $\varphi_{\alpha_{k}}^{-1}(B)$ and $\varphi_{\alpha_{k}}^{-1}(2 B)$ are essentially balls and have comparable volumes (see the remarks at the beginning of $\S 2$ ), it follows from the comments after Lemma (4.2) that $\tilde{u}^{r} \in D^{\infty}$ uniformly in $r$ near 1. The argument for 
$\left\{\tilde{v}^{-1 /(p-1)} \widetilde{\Pi}^{p^{\prime}(n-1)}\right\}^{r}$ is similar:

$$
\begin{aligned}
& \int_{B}\left\{\tilde{v}(t)^{-1 /(p-1)} \tilde{\Pi}^{p^{\prime}(n-1)}\right\}^{r} d t \\
& \approx \int_{\varphi_{n_{k}(B)}^{-1}}\left\{v\left(x+a_{k}\right)^{-1 /(p-1)} \Pi\left(x+a_{k}\right)^{p^{\prime}(n-1)}\right\}^{r} \\
& \cdot(1+|x|)^{\lambda p^{\prime}(n-1) r}|x|^{\alpha_{k} n(1-r)} d x
\end{aligned}
$$

since

and

$$
\widetilde{\Pi}\left(\varphi_{\alpha_{k}}(x)\right) \approx|x|^{-\alpha_{k}}(1+|x|)^{\lambda} \Pi\left(x+a_{k}\right)
$$

$$
\left[-\alpha_{k} n\left(\frac{p}{n}-1\right)\left(\frac{1}{p-1}\right)-\alpha_{k} p^{\prime}(n-1)\right] r+\alpha_{k} n=\alpha_{k} n(1-r) .
$$

This desired doubling condition now follows as above since $\lambda p^{\prime}(n-1) r \geq 0$. This completes the proof of Lemma (4.1).

Remark. In the special case $\lambda=0$, i.e., $\delta=\theta\left(\alpha_{k}+1\right)+\alpha_{k}$, we have $\Pi^{*}=\Pi$ above, and from the fact that (4.4) implies (4.3) we then obtain in case $q=p$ that the condition

$$
\begin{aligned}
& \left(\int_{B} \Pi^{n} d x\right)^{1 / n-1}\left[\left(\int_{B}\left\{\frac{u}{\Pi^{n}}\right\}^{r} \Pi^{n} d x\right)^{1 / r}\left(\int_{B} \Pi^{n} d x\right)^{1 / r^{\prime}}\right]^{1 / p} \\
& \cdot\left[\left(\int_{B}\left\{\frac{v^{-1 /(p-1)} \Pi^{p^{\prime}(n-1)}}{\Pi^{n}}\right\}^{r} \Pi^{n} d x\right)^{1 / r}\left(\int_{B} \Pi^{n} d x\right)^{1 / r^{\prime}}\right]^{1 / p^{\prime}} \leq c
\end{aligned}
$$

implies its analogue for $\tilde{u}, \tilde{v}, \tilde{\Pi}$. This is true without any doubling assumptions, but requires $\lambda=0$. If we use this fact in the proof of Lemma (4.2), we obtain a version of Lemma (4.2) without having to assume any doubling conditionsi.e., without assuming (ii) of Lemma (4.1), provided $\prod_{j=1}^{m}\left(\alpha_{j}+1\right)=\delta+1$. More precisely, we obtain the conclusion of Lemma (4.2) assuming only that (4.5) holds for $\Pi(x)$ as in Lemma (2.13) with $\prod_{j=1}^{m}\left(\alpha_{j}+1\right)=\delta+1$. This leads easily to the following versions of Theorem (1.6) and Corollary (1.8) without doubling assumptions.

Theorem (4.6). Let $\Pi(x)$ be as in Theorem (1.2) except that

$$
\delta=\left[\prod_{j=1}^{m}\left(\alpha_{j}+1\right)\right]-1 .
$$

For $1<p<\infty$, assume that (4.5) holds for some $r>1$ and all balls $B$. Then (1.1) holds with $p=q$ and $c$ independent of $f$.

Corollary (4.7). Let $v(x)$ be as in Corollary (1.4) except that $\gamma+1=$ $p(n-1) \prod_{j=1}^{m}\left[\left\{\beta_{j} / p(n-1)\right\}+1\right]$. For $1<p<\infty$, assume that

$$
|B|^{1 / n}\left[\left(\int_{B}\left\{\frac{u}{\Pi^{n}}\right\}^{r} \Pi^{n} d x\right)^{1 / r}\left(\int_{B} \Pi^{n} d x\right)^{1 / r^{\prime}}\right]^{1 / p} \leq c v(B)^{1 / p}
$$


for some $r>1$ and all balls $B$, where $\Pi(x)$ is defined by

$$
\Pi(x)^{p(n-1)}=(1+|x|)^{\gamma} \prod_{j=1}^{m}\left(\frac{\left|x-a_{j}\right|}{1+\left|x-a_{j}\right|}\right)^{\beta_{j}} .
$$

Then (1.1) holds with $p=q$ and $c$ independent of $f$.

Of course, in verifying Theorem (4.6), it is not necessary to use the part of the argument having to do with $\bar{\Pi}$. In verifying (4.7), we argue as for (1.8) and use the fact that since $\left\{w^{-1 /(p-1)} \Pi^{-n}\right\}^{r} \Pi^{n} \in D^{\infty}$ for $r$ near 1, (2.4) gives

$$
\begin{aligned}
\left(\int_{B}\left\{\frac{w^{-1 /(p-1)}}{\Pi^{n}}\right\}^{r} \Pi^{n} d x\right)^{1 / r} & \left(\int_{B} \Pi^{n} d x\right)^{1 / r^{\prime}} \approx\left(\int_{B} w^{-r /(p-1)} d x\right)^{1 / r}|B|^{1 / r^{\prime}} \\
& \approx \int_{B} w^{-1 /(p-1)} d x
\end{aligned}
$$

for $r$ near 1 since $w^{-1 /(p-1)} \in A_{p^{\prime}}$.

\section{REFERENCES}

1. L. Caffarelli, R. Kohn, and L. Nirenberg, First order interpolation inequalities with weights, Compositio Math. 53 (1984), 259-275.

2. S. Chanillo and R. Wheeden, Weighted Poincaré and Sobolev inequalities and estimates for weighted Peano maximal functions, Amer. J. Math 107 (1985), 1191-1226.

3. E. Fabes, C. Kenig, and R. Serapioni, The local regularity of solutions of degenerate elliptic equations, Comm. Partial Differential Equations 7 (1982), 77-116.

4. A. E. Gatto, C. Gutierrez, and R. Wheeden, Fractional integals on weighted $H^{p}$ spaces, Trans. Amer. Math. Soc. 289 (1985), 575-589.

5. F. Gehring, The $L^{p}$-integrability of the partial derivatives of a quasiconformal mapping, Acta Math. 130 (1973), 265-277.

6. E. Sawyer and R. Wheeden, Weighted inequalities for fractional and Poisson integrals on Euclidean and homogeneous spaces (to appear).

7. J.-O. Strömberg and R. Wheeden, Fractional integrals on weighted $H^{p}$ and $L^{p}$ spaces, Trans. Amer. Math. Soc. 287 (1985), 293-321.

8. J. Vaisala, Lectures on n-dimensional quasiconformal mappings, Lecture Notes in Math., vol. 229, Springer-Verlag, 1971.

Department of Mathematics, DePaul University, Chicago, Illinois 60614

Department of Mathematics, Rutgers University, New Brunswick, New Jersey 08903 